\title{
Prognostic Stratification of Women With Chronic Heart Failure Referred for Heart Transplantation: Relevance of Gender as Compared With Gender-related Characteristics
}

Francesco Grigioni, MD, PhD, ${ }^{\mathrm{a}}$ Andrea Barbieri, MD, ${ }^{\mathrm{b}}$ Antonio Russo, MD, ${ }^{\mathrm{a}}$ Letizia Reggianini, MD, ${ }^{\mathrm{b}}$ Silvia Bonatti, MD, ${ }^{\text {b }}$ Luciano Potena, MD, PhD, ${ }^{\text {a }}$ Francesca Fabbri, MD, ${ }^{\text {a }}$ Fabio Coccolo, MD, ${ }^{\text {a }}$ Gaia Magnani, MD, PhD, ${ }^{\mathrm{a}}$ Francesca Bursi, MD, ${ }^{\mathrm{b}}$ Carlo Magelli, MD, ${ }^{\mathrm{a}}$ Maria G. Modena, MD, ${ }^{\mathrm{b}}$ and Angelo Branzi, MD ${ }^{\mathrm{a}}$

Background: Few studies are available regarding prognostic stratification of women with severe chronic heart failure (CHF). Although women seem to have a better outcome than men, this may be due to favorable baseline characteristics.

Methods: We analyzed a cohort of CHF patients referred for heart transplantation (HT) who underwent clinical/laboratory/instrumental evaluation. Women and men were frequency matched for baseline age $(53 \pm 14$ vs $53 \pm 9$ years, $p=0.92)$, left ventricular ejection fraction $(33 \pm 10$ vs $31 \pm 8 \%$, $p=0.90)$ and ischemic etiology (17 vs $22 \%, p=0.50)$.

Results: $\quad$ A total of 198 patients were analyzed (109 women matched to 89 men). In addition to matching parameters, prevalence of severe symptoms, diabetes and hypertension were also comparable $(p \geq$ 0.25). After 3 years, cardiovascular death or need for HT (CD/HT) event-free survival was $78 \pm 4 \%$ in women and $50 \pm 6 \%$ in men $(p=0.005)$. On multivariate analysis, female gender was associated with a lower risk of CD/HT (relative risk [RR] $0.52 ; 95 \%$ confidence interval [CI] 0.30 to $0.89 ; p=$ $0.017)$, independently of symptoms, blood pressure (BP), left ventricular end-diastolic diameter (LVEDD) and mitral regurgitation (MR). Nevertheless, CD/HT event-free survival at 3 years was $49 \pm 9 \%$ for women with New York Heart Association (NYHA) Class III or IV status, who presented with either severe MR, mean BP $\leq 60 \mathrm{~mm} \mathrm{Hg}$ or LVEDD $\geq 35 \mathrm{~mm} / \mathrm{m}^{2}$.

Conclusions: In advanced CHF, women patients seem to have a better prognosis irrespective of baseline characteristics, supporting the hypothesis that female gender is protective against myocardial injury. However, women with severe symptoms accompanied by either hypotension, severe left ventricular enlargement or MR are at high risk and deserve cautious follow-up and consideration for HT. J Heart Lung Transplant 2006;25:648-52. Copyright (c) 2006 by the International Society for Heart and Lung Transplantation.

Reliable risk stratification in chronic heart failure (CHF) is mandatory to deliver the most appropriate therapeutic strategies, including heart transplantation (HT). Few studies are available to help stratify the prognosis of women with severe $\mathrm{CHF},{ }^{1}$ largely because female patients have been consistently under-represented both in

From the ${ }^{\mathrm{a} C a r d i o l o g y}$ Institute, University of Bologna, Bologna; and

${ }^{\mathrm{b}}$ Cardiology Institute, Modena, Italy.

Submitted August 4, 2005; revised October 10, 2005; accepted February 11, 2006.

Supported by a grant from the University of Bologna donated from the Fondazone Melloni.

Reprint requests: Francesco Grigioni, MD, Istituto di Malattie dell'Apparato Cardiovascolare, Ospedale S. Orsola Malpighi, Via Massarenti 9, 40138 Bologna, Italy. Telephone: XX-39-051-6364526. Fax: XX-39-051-344859. E-mail: francesco.grigioni@unibo.it

Copyright (c) 2006 by the International Society for Heart and Lung Transplantation. 1053-2498/06/\$-see front matter. doi:10.1016/ j.healun.2006.02.004 clinical trials and in studies on prognostic stratification. $^{1,2}$ Most studies ${ }^{1,3-6}$ but not all $^{7}$ indicate that women seem to have a better outcome than men. However, these results may have been influenced by profound gender-related differences in baseline characteristics. ${ }^{8}$ Indeed, women were almost invariably older at baseline, ${ }^{5-7}$ and had (when assessed) better preserved left ventricular ejection fraction (LVEF), ${ }^{3}$ lower prevalence of ischemic etiology $y^{3-7}$ and higher prevalence of diabetes. ${ }^{6,7}$ Furthermore, because the original data included only select clinical/instrumental parameters, ${ }^{1,3-7}$ it was not possible to adjust for the confounding influence of several important predictors of outcome. $^{2,9}$

We analyzed and compared the outcome of women and men within a cohort of CHF patients receiving optimized medical treatment who were referred to the outpatient clinic of our institution, which offers an HT program. Frequency matching was performed accord- 
ing to gender for baseline age, LVEF and prevalence of ischemic etiology to ensure similarity of major determinants of outcome at baseline. Independent prognostic significance of gender was also tested using multivariate analysis.

\section{METHODS \\ Eligibility Criteria and Setting}

All consecutive patients with an established diagnosis of CHF, who were referred to the heart failure clinics of our institutions for prognostic stratification and evaluation for HT from March 1, 1996 through December 31, 2003, were screened for this study. The single eligibility criterion was concomitant availability at index evaluation of a clinical, laboratory, electrocardiographic (ECG) and echocardiographic assessment. To increase the statistical power of the study, ${ }^{10}$ all eligible women were matched to eligible men for 3 strong confounders: age (baseline); LVEF (baseline); and underlying ischemic etiology of CHF (as confirmed at coronary angiography). Because the matching process involved multiple variables, frequency matching was selected. ${ }^{11,12}$ The computerized matching process was conducted in a fully blinded fashion (and done before any outcome information was obtained). A 2-dimensional standard transthoracic echocardiogram (Sonos 5500, Hewlett Packard) was performed as previously described. ${ }^{13}$ Severity of mitral regurgitation was evaluated semiquantitatively from the area of the regurgitant jet by color Doppler assessment. ${ }^{14}$ All patients had provided written consent to confidential use of their data. The study was conducted in accordance with our institutional guidelines and national legal requirements.

\section{Statistical Analysis}

Continuous variables are expressed as mean $\pm \mathrm{SD}$ or as otherwise reported, and categoric variables as numbers (percentages). Group comparisons were performed using the $t$-test or chi-square test, as appropriate. The combined end-point was cardiovascular death or need for heart transplantation $(\mathrm{CD} / \mathrm{HT})^{15-21}$; analysis was performed with censoring at the time of cardiac surgery (other than HT), whenever this was performed $(n=1)$. Event rates were estimated using the Kaplan-Meier method. Each baseline variable was individually tested by univariate Cox proportional hazards analysis (because frequency matching was necessarily conducted using categoric definitions of LVEF and age, these 2 parameters were also tested as continuous variables). ${ }^{10}$ Variables that reached $p<0.10$ were then used to create a multivariate model. The variable "gender" was then added into this model. $p<0.05$ was considered statistically significant. All tests were performed using
Table 1. Principal Baseline Characteristics of the Study Population According to Gender

\begin{tabular}{lccc}
\hline & $\begin{array}{c}\text { Women } \\
(n=109)\end{array}$ & $\begin{array}{c}\text { Men } \\
(n=89)\end{array}$ & $p$ \\
\hline Age (years) & $53 \pm 14$ & $53 \pm 9$ & 0.92 \\
NYHA Class III or IV & $38(35 \%)$ & $33(37 \%)$ & 0.77 \\
Ischemic etiology of & & & \\
$\quad$ congestive heart failure & $19(17 \%)$ & $20(22 \%)$ & 0.50 \\
Mean blood pressure (mm Hg) & $57 \pm 6$ & $56 \pm 9$ & 0.65 \\
Hypertension & $49(45 \%)$ & $32(36 \%)$ & 0.25 \\
Diabetes & $18(16 \%)$ & $13(15 \%)$ & 0.85 \\
Atrial fibrillation & $9(8 \%)$ & $14(16 \%)$ & 0.12 \\
QRS duration (ms) & $136 \pm 37$ & $140 \pm 38$ & 0.37 \\
Left atrial diameter (mm/m $\left.{ }^{2}\right)$ & $28 \pm 7$ & $26 \pm 5$ & 0.053 \\
Left ventricular end-diastolic & & & \\
$\quad$ diameter (mm/m $\left.{ }^{2}\right)$ & $38 \pm 5$ & $38 \pm 7$ & 0.60 \\
Left ventricular end-systolic & & & \\
$\quad$ diameter (mm/m $\left.{ }^{2}\right)$ & $31 \pm 6$ & $33 \pm 7$ & 0.082 \\
Left ventricular ejection & & & \\
$\quad$ fraction (\%) & $33 \pm 10$ & $31 \pm 8$ & 0.90 \\
Severe mitral regurgitation & $37(34 \%)$ & $33(37 \%)$ & 0.66 \\
\hline
\end{tabular}

STATVIEW 5.0.1 software (SAS Institute, Inc.) for Windows.

\section{RESULTS Study Population}

A total of 198 patients entered the analysis (all 109 eligible women, frequency matched to 89 eligible men). Table 1 indicates the main baseline characteristics of the study population: these major determinants of prognosis appeared mostly comparable in men and women. The impairment in clinical/instrumental parameters indicates that $\mathrm{CHF}$ was generally severe. The young mean age likely reflects the institutional availability of an HT program. With regard to medications, 87 $(80 \%)$ women and $70(79 \%)$ men were taking betablockers $(\phi=0.90)$; angiotensin-converting enzyme (ACE) inhibitors were taken by 96 (88\%) female patients and by $83(93 \%)$ male patients $(p=0.24)$. The 2 most common ACE inhibitors used were enalapril (66 patients; mean dosages in women and men: $15 \pm 7$ vs $19 \pm 15 \mathrm{mg} / \mathrm{day} ; p=0.19$ ) and ramipril (43 patients; mean dosages in women and men: $6 \pm 3$ vs $5 \pm 2$ $\mathrm{mg} /$ day; $p=0.21$ ). Eighty-seven (80\%) women and 70 (79\%) men were taking beta-blockers $(p=0.90)$. The most common beta-blocker prescribed was carvedilol (138 patients; dosages in women and men: $25 \pm 16 \mathrm{vs}$ $22 \pm 17 \mathrm{mg} / \mathrm{day} ; p=0.15)$. Use of implantable cardioverter defibrillators appeared comparable in men $(n=$ 11) and women $(n=8)(p=0.33)$.

\section{Incidence of Adverse Cardiac Events According to Gender}

During a mean medical follow-up of $24 \pm 18$ months (in $99 \%$ of patients followed up to 2004 or death), 17 


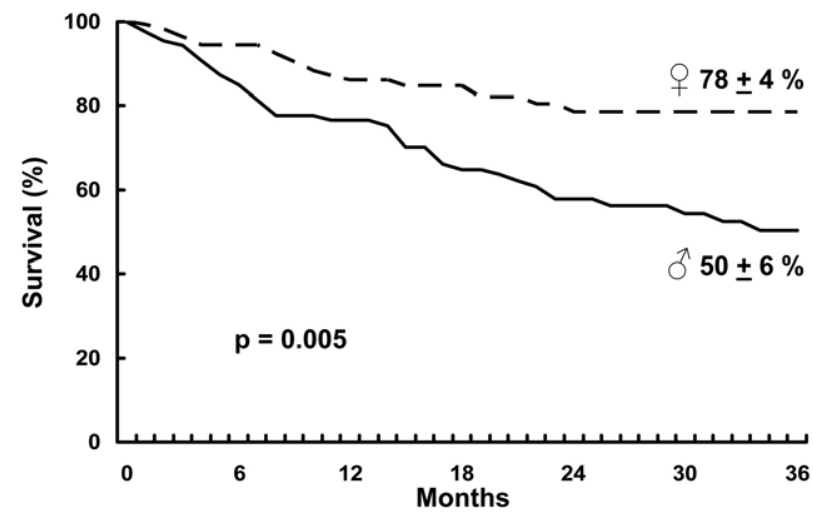

Figure 1. CD/HT event-free survival according to gender among 198 CHF patients with similar baseline characteristics.

patients died from cardiovascular death (CD) and a further 47 required HT due to worsening clinical conditions. Cardiovascular deaths were attributed to $\mathrm{CHF}$ in 13 of $17(76 \%)$ patients. Ten (58\%) of the deaths were sudden. Figure 1 shows CD/HT event-free survival curves according to gender. $\mathrm{CD} / \mathrm{HT}$ event-free survival at 3 years turned out to be significantly more favorable in women ( $78 \pm 4 \%$ vs $50 \pm 6 \% ; p=0.005)$, although the matching process (for age, LVEF and ischemic etiology) led to similarities in most other major baseline characteristics.

\section{Analysis of Predictors of Adverse Cardiac Events}

Non-gender-related predictors. The following variables reached $p<0.1$ according to univariate analysis, with CD/HT as the end-point: New York Heart Association (NYHA) Class III or IV status $(p<0.0001)$; mean blood pressure (BP; $p<0.0001)$; QRS duration $(p=$ 0.009); left ventricular end-systolic and end-diastolic diameter (LVEDD); LVEF; left atrial diameter; and presence of severe functional mitral regurgitation (MR) (all $p<0.001$ ). Non-gender-related variables that turned out to be independent predictors of $\mathrm{CD} / \mathrm{HT}$ according to multivariate analysis are reported in Table 2 .

Effect of gender. On univariate analysis, the risk ratio (RR) for female gender was 0.49 , with a $95 \%$ confidence interval $(95 \% \mathrm{CI})$ of 0.29 to $0.81(p=0.006)$. Table 3 reports multivariate predictors of $\mathrm{CD} / \mathrm{HT}$ after female

Table 2. Non-gender-related Predictors of CD/HT at Multivariate Analysis

\begin{tabular}{lccc}
\hline & $\begin{array}{c}\text { Risk } \\
\text { ratio }^{\mathrm{a}}\end{array}$ & $\begin{array}{c}95 \% \text { confidence } \\
\text { interval }\end{array}$ & $p$ \\
\hline NYHA Class III or IV & 2.80 & $1.58-4.97$ & $<0.001$ \\
Mean blood pressure $(\mathrm{mm} \mathrm{Hg})$ & 0.94 & $0.91-0.97$ & $<0.001$ \\
$\begin{array}{l}\text { Left ventricular end-diastolic } \\
\quad \text { diameter (mm/m }\end{array}$ & & & \\
Severe mitral regurgitation & 1.07 & $1.03-1.12$ & 0.002 \\
\hline a & 1.77 & $1.00-3.12$ & 0.051 \\
\hline
\end{tabular}

${ }^{a}$ Expressed per unit of each determinant.
Table 3. Multivariate Predictors of $\mathrm{CD} / \mathrm{HT}$ Factoring the Variable "Gender" Into the Model

\begin{tabular}{lccr}
\hline & $\begin{array}{c}\text { Risk } \\
\text { ratio }^{\mathrm{a}}\end{array}$ & $\begin{array}{c}95 \% \text { confidence } \\
\text { interval }\end{array}$ & \multicolumn{1}{c}{$p$} \\
\hline NYHA Class III or IV & 3.04 & $1.72-5.38$ & $<0.001$ \\
Mean blood pressure $(\mathrm{mm} \mathrm{Hg})$ & 0.95 & $0.92-0.98$ & 0.002 \\
Left ventricular end-diastolic & & & \\
$\quad$ diameter (mm $/ \mathrm{m}^{2}$ ) & 1.06 & $1.02-1.11$ & 0.004 \\
Severe mitral regurgitation & 1.96 & $1.11-3.47$ & 0.020 \\
Female gender & 0.52 & $0.30-0.89$ & 0.017 \\
\hline
\end{tabular}

${ }^{a}$ Expressed per unit of each determinant.

gender was factored into the multivariate Cox proportional hazards model. Female gender was independently associated with a reduced risk of adverse cardiac events (RR $0.52 ; 95 \%$ CI 0.30 to $0.89 ; p=0.017$ ). The independent prognostic significance of female gender was retained even when the following variables were individually forced into the model: presence of multivessel disease by coronary angiography $(n=25$; RR $0.46 ; p=0.018)$; age (RR $0.53 ; p=0.022$ ); and LVEF (RR $0.50 ; p=0.013$ ). CD/HT event-free survival at 3 years in women with NYHA Class III or IV status, with either severe MR, mean $\mathrm{BP} \leq 60 \mathrm{~mm} \mathrm{Hg}$ (corresponding to the approximation of the median value of mean $\mathrm{BP}$ ) or LVEDD $\geq 35 \mathrm{~mm} / \mathrm{m}^{2}$ (corresponding to the to the approximation of the median value of LVEDD), was $49 \pm 9 \%$ ( $n=37 ; p<0.001$ vs the other women). Notably, the use of ACE inhibitors was not significantly different between female patients with and without mean $\mathrm{BP} \leq 60 \mathrm{~mm} \mathrm{Hg}(p=0.13)$.

\section{DISCUSSION}

Limited data are available to help stratify the prognosis of female patients with CHF. ${ }^{1}$ Furthermore, it remains unclear whether the reportedly more favorable outcome of women is related to gender per se or depends on favorable baseline characteristics. ${ }^{9,22,23}$ The present study was specifically designed not only to help prognostic stratification of female CHF patients, but also to analyze, for the first time, the influence of gender in the presence of largely comparable baseline characteristics.

All the patients had been submitted to a very extensive set of concomitant clinical, instrumental and laboratory assessments. ${ }^{2,9}$ To increase the statistical power of the study, ${ }^{10}$ we adopted frequency matching for 3 major determinants of outcome. This decision allowed us to take into account the possible influence of a wide range of possible confounders. ${ }^{10-12}$ Despite largely similar medical treatment and baseline values for a series of prognostically relevant variables ${ }^{9}$ (Table 1 ), the women had a better outcome in terms of a lower incidence of major adverse cardiac events (Figure 1). Moreover, gender retained independent prognostic significance for these adverse events even after adjusting 
at multivariate analysis for all of the independent predictors of outcome deriving from the comprehensive set of clinical, instrumental and laboratory variables.

Evidence of better survival among women (derived from analyses of populations with heterogeneous baseline characteristics) has often been explained in terms of their higher mean baseline values of LVEF. ${ }^{8}$ Notably, the Framingham study ${ }^{24}$ and NHANES 1 study,$^{25}$ neither of which assessed LVEF, showed more favorable survival in women with CHF. However, these findings were not confirmed in the SOLVD study, which enrolled only patients with established low $\mathrm{LVEF}^{7}$ More recent reports ${ }^{1,5,6}$ supporting a protective effective of female gender involved disputable adjustments for major differences at baseline-including LVEF, age and ischemic etiology-sometimes in the presence of a different medical treatment. It has been hypothesized that gender could influence the pathophysiologic response to myocardial injury. Clinical and biologic studies have indicated that a protective effect of female gender could be due to a more favorable response of the left ventricle to pressure overload, ${ }^{26}$ attenuated sympathetic activation and parasympathetic withdrawal, ${ }^{27}$ reduced myocyte necrosis and apoptosis ${ }^{28}$ or a protective effect of gender hormones on the renin-angiotensin system. ${ }^{29}$

In the present study, NYHA class, BP, LVEDD and presence of severe functional MR turned out to be independent predictors of serious adverse cardiac events even when adjusted for gender. While providing useful confirmation of the prognostic value of some previously identified predictors of outcome, ${ }^{1,2}$ our results suggest a novel combination of commonly available indicators of prognosis, which may be useful for prognostic stratification of female patients. Notably, female patients who presented with severe symptoms associated with either hypotension, severe left ventricular enlargement or MR turned out to be at high risk, with an event-free survival at 3 years comparable to male patients. Taken together these findings indicate that female patients presenting these factors are at high risk and therefore deserve cautious follow-up. Of note, the broad similarity of use of ACE inhibitors in women with and without mean $\mathrm{BP} \leq 60 \mathrm{~mm} \mathrm{Hg}$ suggests that outcome differences were more likely due to the underlying severity of CHF rather than to beneficial effects of this class of drugs. ${ }^{2}$

Regarding study limitations, in this retrospective analysis of prospectively collected data, $\mathrm{CD}$ and need for $\mathrm{HT}$ were considered as a combined end-point-a highly relevant clinical outcome measure that has been used widely in previous studies on $\mathrm{CHF} .{ }^{15-21}$ Furthermore, even when the analysis was repeated censoring the follow-up at the time of HT and considering acute heart failure or $\mathrm{CD}$ as the end-point, the RR related to female gender remained similar (RR $0.40, p=0.002$ ). The clinical/instrumental assessment was obtained at a given timepoint, and it is possible that studies analyzing time-related changes in prognostic parameters could provide a further relevant contribution to the topic of gender and $\mathrm{CHF} .{ }^{21}$ Unfortunately, information on the duration of CHF was not available.

Although prevalence of ischemic heart disease was similar in men and women $(p=0.50)$, the event-free survival was more favorable in female patients (Figure 1). Furthermore, the lower risk of adverse events that characterized female gender was confirmed on multivariate analysis independently from the presence of ischemic etiology of CHF (RR $0.52, p=0.018$ ). Taken together, these findings suggest that the survival advantage of female gender is not affected by the presence of coronary artery disease, although the relatively low prevalence of CHF due to ischemic heart disease in the present series does not allow firm conclusions with respect to this issue. ${ }^{1}$

In conclusion, in advanced CHF, female patients seem to have a better prognosis irrespective of baseline characteristics, supporting the hypothesis that female gender exerts a protective effect against myocardial injury. Nevertheless, women with severe symptoms and either hypotension, severe left ventricular enlargement or MR are at high risk and deserve cautious follow-up and consideration for HT.

The authors are grateful to Robin M. T. Cooke for assistance with manuscript preparation.

\section{REFERENCES}

1. Ghali JK, Krause-Steinrauf HJ, Adams KF, et al. Gender differences in advanced heart failure: insights from the BEST study. JAMA 2003;42:2128-34

2. Aaronson KD, Schwartz JS, Chen TM, et al. Development and prospective validation of a clinical index to predict survival in ambulatory patients referred for cardiac transplant evaluation. Circulation 1997;95:2660-7.

3. Adams KF Jr, Dunlap SH, Sueta CA, et al. Relation between gender, etiology and survival in patients with symptomatic heart failure. JAMA 1996;28:1781-8.

4. Adams KF Jr, Sueta CA, Gheorghiade M, et al. Gender differences in survival in advanced heart failure. Insights from the FIRST study. Circulation 1999;99:1816-21.

5. Simon T, Mary-Krause M, Funck-Brentano C, Jaillon P. Sex differences in the prognosis of congestive heart failure: results from the Cardiac Insufficiency Bisoprolol Study (CIBIS II). Circulation 2001;103:375-80.

6. Ghali JK, Pina IL, Gottlieb SS, Deedwania PC, Wikstrand JC. Metoprolol CR/XL in female patients with heart failure: analysis of the experience in Metoprolol Extended-Release Randomized Intervention Trial in Heart Failure (MERIT-HF). Circulation 2002;105:1585-91.

7. Bourassa MG, Gurne O, Bangdiwala SI, et al. Natural history and patterns of current practice in heart failure. 
The Studies of Left Ventricular Dysfunction (SOLVD) Investigators. JAMA 1993;22:14A-9.

8. Jessup M, Pina IL. Is it important to examine gender differences in the epidemiology and outcome of severe heart failure? J Thorac Cardiovasc Surg 2004;127:1247-52.

9. Cowburn PJ, Cleland JG, Coats AJ, Komajda M. Risk stratification in chronic heart failure. Eur Heart J 1998;19: $696-710$.

10. Szklo M, Nieto J. Epidemiology beyond the basics. Sudbury, MA: Jones and Bartlett; 2004.

11. Samuels ML. Matching and design efficiency in epidemiological study. Biometrika 1981;68:577-88.

12. Thompson WD, Kelsey JL, Walter SD. Cost and efficiency in the choice of matched and unmatched case-control study designs. Am J Epidemiol 1982;116:140-51.

13. Tajik AJ, Seward JB, Hagler DJ, Mair DD, Lie JT. Twodimensional real-time ultrasonic imaging of the heart and great vessels. Technique, image orientation, structure identification, and validation. Mayo Clin Proc 1978;53:271-303.

14. Bursi F, Enriquez-Sarano M, Nkomo VT, et al. Heart failure and death after myocardial infarction in the community: the emerging role of mitral regurgitation. Circulation 2005; 111:295-301.

15. Stevenson LW, Steimle AE, Fonarow G, et al. Improvement in exercise capacity of candidates awaiting heart transplantation. JAMA 1995;25:163-70.

16. Fauchier L, Babuty D, Cosnay P, Autret ML, Fauchier JP. Heart rate variability in idiopathic dilated cardiomyopathy: characteristics and prognostic value. JAMA 1997;30: 1009-14.

17. Gullestad L, Myers J, Ross H, et al. Serial exercise testing and prognosis in selected patients considered for cardiac transplantation. Am Heart J 1998;135:221-9.

18. Florea VG, Henein MY, Anker SD, et al. Prognostic value of changes over time in exercise capacity and echocardiographic measurements in patients with chronic heart failure. Eur Heart J 2000;21:146-53.
19. Grigioni F, Carinci V, Boriani G, et al. Accelerated QRS widening as an independent predictor of cardiac death or of the need for heart transplantation in patients with congestive heart failure. J Heart Lung Transplant 2002;21: 899-902.

20. Peterson LR, Schechtman KB, Ewald GA, et al. The effect of beta-adrenergic blockers on the prognostic value of peak exercise oxygen uptake in patients with heart failure. J Heart Lung Transplant 2003;22:70-7.

21. Grigioni F, Barbieri A, Magnani G, et al. Serial versus isolated assessment of clinical and instrumental parameters in heart failure: prognostic and therapeutic implications. Am Heart J 2003;146:298-303.

22. Wenger NK. Women, heart failure, and heart failure therapies. Circulation 2002;105:1526-8.

23. Pina IL. A better survival for women with heart failure? It's not so simple. JAMA 2003;42:2135-8.

24. Ho KK, Anderson KM, Kannel WB, Grossman W, Levy D. Survival after the onset of congestive heart failure in Framingham Heart Study subjects. Circulation 1993;88: 107-15.

25. Schocken DD, Arrieta MI, Leaverton PE, Ross EA. Prevalence and mortality rate of congestive heart failure in the United States. JAMA 1992;20:301-6.

26. Mendes LA, Davidoff R, Cupples LA, Ryan TJ, Jacobs AK. Congestive heart failure in patients with coronary artery disease: the gender paradox. Am Heart J 1997;134:20712 .

27. Aronson D, Burger AJ. Gender-related differences in modulation of heart rate in patients with congestive heart failure. J Cardiovasc Electrophysiol 2000;11:1071-7.

28. Guerra S, Leri A, Wang X, et al. Myocyte death in the failing human heart is gender dependent. Circ Res 1999; 85:856- 66 .

29. Fischer M, Baessler A, Schunkert H. Renin angiotensin system and gender differences in the cardiovascular system. Cardiovasc Res 2002;53:672-7. 